\title{
El olvido en la justicia digital: un derecho fundamental autónomo ${ }^{1}$
} Forgetting in digital justice:
a fundamental autonomous right

María Fernanda Cerón Gracia ${ }^{2}$

REC: 09/12/2020 STEPHANY DORADO SANDOVAL ${ }^{3}$

Alex Rodrigo ColL ${ }^{4}$

Rubén DARío Restrepo Rodríguez ${ }^{5}$

\section{Resumen}

La información sensible de las personas, contenida y gestionada a través de dispositivos y mecanismos tecnológicos, ha generado una latente tensión entre varios derechos fundamentales como el acceso a la justicia y la intimidad, porque no se vislumbra una efigie jurídica que establezca límites concretos cuando aquellos entran en contacto. Con el presente artículo se pretende, en esa medida, demostrar que en la era digital - y de manera más específica, en la justicia digital- la información sensible de las personas exige un halo de protección autónomo y fundamental que no está presente en la conexidad como apuesta teórica actual y que, además, dicha categoría diezma la esfera de protección presente en esta prerrogativa que sí se concibe como un derecho fundamental autónomo.

1. Artículo resultado del proyecto de investigación titulado "Justicia asertiva, litigio digital y asimetrías geo-sociales. Relación efectivismo-eficientismo de la gestión judicial", liderado por los docentes Alex Rodrigo Coll y Rubén Darío Restrepo Rodríguez, financiado por la Fundación Universitaria Católica Lumen Gentium.

2. Estudiante de octavo semestre del Programa de Derecho de la Fundación Universitaria Católica Lumen Gentium. Integrante del Semillero de Investigación en Derecho Público y Derecho de Interés Público (SIDEP - Grupo de Investigación en Derecho y Ciencias Políticas). Correo electrónico: maria.ceron01@unicatolica.edu.co

3. Estudiante de cuarto semestre del Programa de Derecho de la Fundación Universitaria Católica Lumen Gentium. Integrante del Semillero de Investigación en Derecho Público y Derecho de Interés Público (SIDEP - Grupo de Investigación en Derecho y Ciencias Políticas). Correo electrónico: stephany.dorado01@unicatolica.edu.co

4. Abogado y Magíster en Derecho. Docente del Departamento de Ciencias Jurídicas y Políticas de la Fundación Universitaria Católica Lumen Gentium y líder del Grupo de Investigación en Derecho y Ciencias Políticas. Correo electrónico: acoll@unicatólica.edu.co. Orcid: https:// orcid.org/0000-0001-7294-0702

5. Abogado y Magíster en Derecho. Docente del Departamento de Ciencias Jurídicas y Políticas de la Fundación Universitaria Católica Lumen Gentium y líder del Grupo de Investigación en Derecho y Ciencias Políticas. Correo electrónico: rdrestrepo@unicatolica.edu.co. Orcid: https:// orcid.org/0000-0001-8238-6944 
Para ello se partió de la teoría de los derechos fundamentales, afincada desde la autonomía como característica valiosa, de cara a concretar la exigibilidad de tales prerrogativas jurídicas en cualquiera de los contextos de tensión.

Palabras clave: Derecho al olvido, justicia digital, derechos fundamentales, autonomía.

\section{Abstract}

Sensitive information from people contained and managed through technological devices and mechanisms has generated a latent tension between some fundamental rights such as access to justice and privacy, because there is no legal effigy that sets concrete limits when those come into contact. This article aims, to that extent, to demonstrate that in the digital age - and more specifically in digital justice-, sensitive information of people requires a halo of autonomous and fundamental protection that is not present in the related as a current theoretical bet and that, moreover, this category decimates the area of protection present in this prerogative if it is conceived as an autonomous fundamental right. This was done from the theory of fundamental rights, which was fundamentality from autonomy, as a valuable feature in order to concretize the enforceability of such legal prerogatives in any of the contexts of tension.

Keywords: Right to be forgotten, digital justice, fundamental rights, autonomy.

\section{Introducción}

La implementación de las tecnologías ha generado cambios en la vida humana, de diversas formas; entre esas transformaciones, una de las más visibles es la facilitación comunicacional y el minimalismo en los instru- mentos, herramientas y dispositivos electrónicos. En el campo de la función pública de impartición de justicia, la presencia de las tecnologías ha permitido la inmaterialización del dossier y la dilución de barreras geográficas a través de la litigación digital, además de las bondades en las actividades administrativas propias de los despachos judiciales. No obstante, surgen inconvenientes respecto del derecho al olvido y el derecho a la intimidad, debido al aperturismo de la información sensible que se ventila en los procesos y que ahora reposa en archivos digitales y, por lo tanto, disponible en la red.

La investigación propone las bases conceptuales para el fortalecimiento de un marco teórico que sustente al olvido como un derecho fundamental y autónomo, siendo la problemática que genera la transición de la justicia desde su concepción bajo el uso de las tecnologías que modifican la práctica administrativa judicial. Lo anterior se realiza bajo el método de exploración documental de corte teórico básico; se hace el análisis de los referentes bibliográficos de diferentes autores tales como Mieres (2014), Leturia (2016), Londoño (2010), Ferrajoli (2006), Coll (2018), Coll y González (2020) y Chéliz (2016), citados para la conceptualización de la fundamentalidad y autonomía del derecho al olvido como una garantía a los intervinientes procesales. Se parte de conceptos predominantes como los derechos fundamentales, la autonomía, el derecho al olvido, la intimidad y la justicia digital.

\section{El olvido como derecho fundamental}

\section{Derechos fundamentales}

Los derechos fundamentales se han comprendido desde diferentes perspectivas, a partir de las cuales se han establecido los 
criterios para su identificación y clasificación. Para Marshall (2017), "son aquellos que tienen un sentido calificativo, que han sido determinados a ciertos derechos sin los cuales la existencia de un orden estatal sería ilegítima. Sería un conjunto de derechos de los cuales pendería la legitimación del orden jurídico-estatal" (p. 97). Es decir, son aquellos derechos humanos que gozan de una afirmación por el ordenamiento jurídico desde la Constitución como un reconocimiento propio del derecho positivo interno. Arango (2005) afirma que "este debe tener una característica importante y subjetiva, que ostenta garantías de reconocimiento o positivización por parte del Estado, con obligatoriedad de cumplimiento y recaiga sobre el sujeto" (p. 32). A la par, en la Sentencia T-1306/00, la Corte Constitucional sostuvo que "son criterios de derechos constitucionales fundamentales, los de la persona humana y los de reconocimientos expresos. Es decir, el primero hace referencia a la base material y el segundo a lo formal". Los derechos fundamentales no solo se limitan a los reconocidos por el ordenamiento jurídico, sino que se extienden también a los que son parte de los catálogos axiológicos que garantizan la dignidad humana.

En una sociedad que muta de forma permanente, arrastrada por los vertiginosos avances tecnológicos, las taxonomías no representan un elemento dinamizador de los derechos sino un cimiento de la arquitectura garantista de aquellos. La introducción de otros criterios para el reconocimiento de nuevos derechos permite a las sociedades mantener su condición de humanidad sobre la acumulación, el poder $y$, entre otros, el desarrollismo tecnológico, que se cuelan en la intersubjetividad como elementos axiales, diluyendo su instrumentalidad frente a la relevancia humana y pareciera que reclaman protagonismo. Para Coll (2018), "los derechos de las personas y en especial los que le son inherentes a estas, ya no son considerados como asuntos exóticos y extraños al universo jurídico y político" (p. 82). Su fundamentalidad no radica limitativamente en criterios de reconocimiento atados al legiscentrismo jurídico estipulados de manera expresa en la Constitución, por lo que establece que por ser inherentes a la persona gozarán de la misma importancia, delimitando el uso y goce de catálogos axiológicos y designándolos como derechos fundamentales nominados e innominados.

Los nominados son los que gozan de un reconocimiento por parte del ordenamiento jurídico de manera interna, taxativamente en la Constitución (Ferrajoli, 2006, pp. 116, 117). Por otro lado, los innominados "son aquellos derechos que, si bien se encuentran implícitos en el ámbito de protección de distintas disposiciones jurídicas fundamentales, no se encuentran textualmente enunciados en ellas" (Garzón, 2016, p. 21). Sin embargo, la falta de enunciación taxativa no es una negación de su existencia ni de su contribución a otros derechos fundamentales, conformando un sistema jurídico protector complejo que va más allá del texto normativo. De igual forma se deben garantizar por ser inherentes a la condición del ser (Coll, 2018, p. 82), estableciendo límites y vínculos que el Estado deberá asumir como garante frente a los elementos que la amenazan, la disminuyen o la niegan de forma total. De allí que la consolidación de nuevos derechos fundamentales recaiga en el desarrollo académico, judicial y político que los actores del sistema generen, los cuales ingresan de forma implícita a la gama de derechos constitucionales.

Resulta importante, entonces, recabar sobre los alcances que contiene la categoría de derechos fundamentales. Por una parte, representan la máxima expresión jurídica para garantizar la realización del individuo como impronta de su libertad. Por otra parte, 
contribuyen a la consolidación de las sociedades en la medida que viabilizan la interacción en climas de aproximación hacia la plausible idea de justicia que se reconoce y se acepta en cada comunidad. A la par, su reflejo en otros agregados sociales persigue fines de réplica 0 de corrección. Esta multidimensionalidad de los derechos fundamentales en las relaciones intersubjetivas, exige que su reconocimiento, clasificación y defensa evolucione en la necesidad y medida de las transformaciones sociales, en especial, a la velocidad que imprime la emergente comunidad que de Lucas (2008) distingue con el epíteto de "sociedad reticular" (p. 9).

Sin embargo, los anteriores criterios de reconocimiento de los derechos fundamentales - los nominados y los innominados- se complementan con el de la conexidad, que permite que un derecho no fundamental sea susceptible de tratamiento y protección bajo dicha categoría ante la actual o inminente afectación negativa colateral a un derecho fundamental. Como sucedía otrora con el derecho a la salud y a la vida, donde el primero de los mencionados no contaba con la categoría de fundamental pero que, una vez dada su afectación, los efectos negativos se extendían al derecho fundamental a la vida y, por lo tanto, afincada, por la vía de la conexidad, una posición ius-fundamental.

Si bien, la jurisprudencia constitucional ha señalado en múltiples ocasiones que el derecho a la salud no es en sí mismo un derecho fundamental, también le ha reconocido amparo de tutela en virtud de su conexidad con el derecho a la vida y con la integridad de la persona, en eventos en que deslindar salud y vida es imposible y se hace necesario asegurar y proteger al hombre y su dignidad. Por esta razón, el derecho a la salud no puede ser considerado en sí mismo como un derecho autónomo y fundamental, sino que deriva su protección inmediata del vínculo inescindible con el derecho a la vida. (Corte Constitucional, 1998)
A pesar del aparente favorecimiento que generaba el criterio de la conexidad, subrepticiamente justificaba la no incorporación de la salud como un derecho fundamental y por contera de su autonomía. La autonomía se traduce en la capacidad de acción sin consideración a determinismos externos que la diluyan o la enerven por completo. Esta definición se logra colegir a partir de los planteamientos, entre otros, de Moreno y Esponda (2008), quienes afirman que "la autonomía sería, pues, el principio supremo que posibilita auténticas acciones morales" (p. 9). En el mismo sentido, para Kant (1875), la autonomía es "el estado mediante el cual una ley es para sí misma, independiente de cómo están constituidos los objetos del querer" (p. 22). Es decir, la elección que se da para la toma de decisiones propias, sin vínculo exterior para su aplicabilidad. En el ámbito jurídico, la noción de autonomía se debe capitalizar para garantizar que los derechos y en especial los fundamentales, sean verdaderos mecanismos de protección para la dignidad humana.

Hoy se muestra artificioso predicar la exigencia de conexidad respecto de derechos fundamentales los cuales tienen todos - unos más que otros- una connotación prestacional innegable. Ese requerimiento debe entenderse en otros términos, es decir, en tanto enlace estrecho entre un conjunto de circunstancias que se presentan en el caso concreto. (Corte Constitucional, 2007, 2008)

La autonomía delimita la tendencia subrepticia de desconocerlos, de amenazarlos y de vulnerarlos, que se arraiga en teoremas de regateo político y económico con pilares como los derechos programáticos y otras por la vía de la conexidad. Esta última, si bien es cierto suele ser beneficiosa para amplificar la protección de algunos derechos no fundamentales, también lo es, que se trata de un potencial reductor de aquellos derechos que 
en esencia son inherentes a la condición de humanidad pero que ante su no positivización mantienen halos de incertidumbre jurídica 0 comportan garantías más tenues que aquellas que su importancia exige. La autonomía en los derechos fundamentales es una característica definitoria y tiende vínculos ligados al cuidado y protección de la integridad del individuo que redunda en la exigibilidad social, administrativa y judicial.

\section{Derecho al olvido}

El derecho al olvido es la facultad que tiene el individuo para que la información privada suministrada en las diferentes bases de datos sea, en su más extenso alcance de protección, eliminada 0 anonimizada y en sus alcances de mínima protección, se establezcan límites en el uso y tratamiento de aquella cuando su eliminación no se presenta como la mejor de las alternativas de solución. Mieres (2014) afirma que "el olvido es la última manifestación de la necesidad de preservar la privacidad de las personas frente a las amenazas que entraña el progreso tecnológico" (p. 6). Es decir que, su fin radica en proteger la integridad y la libertad para el desarrollo del individuo, dando cabida a un entorno social libre de prejuicios por parte de la sociedad. Brinda seguridad en el uso y goce de otros derechos necesarios sustanciales del individuo como parte intrínseca de la dignidad humana. Su uso no solo recae sobre la gestión de la información, sino que, a la par, resguarda diversas prerrogativas individuales como el buen nombre, la intimidad, la honra y la imagen, entre otras, que, contando o no con un mecanismo de acción jurisdiccional propio, son objeto de permanente amenaza, vulneración o desconocimiento, dado el estrecho vínculo que su estructura genética guarda con la gestión de la información sensible que se aloja en la red 0 en las bases de almacenamiento.
Lo anterior no sugiere que el olvido se constituya en una prerrogativa inmune y absoluta ante la tensión o contacto con otros derechos y objetos de protección jurídica, sino que surge de la proclama que la condición humana presenta, sea de forma tácita 0 expresa, frente a las arrolladoras transformaciones que incorpora el avance tecnológico en la vida de las personas. El olvido como un derecho fundamental no sugiere la eliminación 0 anonimización de la información con único fundamento en el ejercicio del derecho de acción por parte de su titular. Exige del operador, intérprete 0 autoridad, que se conjugue con la oposición que presentan otros derechos y otros fines que se persiguen en cada actuación, de forma que no exista una libertad absoluta en el manejo de la información sensible, empero que tampoco, el olvido se constituya en una talanquera insalvable que impida la consecución de otros fines que, según las particulares condiciones de cada situación, reclamen desde el ejercicio racional, una prelación o al menos un uso delimitado de la información sensible al uso abierto.

Las garantías de protección a la privacidad, con el paso a la sociedad tecnológica, exigen establecer criterios que hagan posible su fin, basándose en "el derecho de cancelación, y el derecho de oposición" (Chéliz, 2016, p. 257). El derecho de cancelación para Guasch y Soler (2015) se presenta en "aquellas situaciones donde los datos se han tratado de forma legítima con un fin y de la misma manera dejan de tener validez para respetar el principio de calidad procediendo a su cancelación por ser inexactos o incompletos" (p. 995) y hasta innecesarios o excesivos.

El derecho de oposición, según Guasch y Soler (2015), "es el derecho del afectado a que no se lleve a cabo el tratamiento de sus datos de carácter personal o se cese en el 
mismo" (p. 993). El enfoque principal del derecho al olvido es garantizar y proteger el libre desarrollo de la persona, con limitaciones al uso de la información privada en el presente 0 en el futuro, impidiendo que la información sensible de las personas afecte su entorno y desenvolvimiento social o se convierta en un obstáculo insalvable de la incorporación de estas en aquella. Es una ruptura de las cadenas y condenas sociales que inhiben el aprovechamiento de nuevas oportunidades en la vida o de liberar las cargas de un pasado indeseado por cualquiera que fuere la razón de su inoportunidad futura. Opera de la misma forma para resguardar aquella información que pertenece a la esfera dependiente del poder dispositivo de las personas: la intimidad.

La intimidad compromete todo aquello que se encuentra inmerso en el ámbito más propio y oculto del ser humano - entendiéndose por propio y oculto, la información que se mantiene para sí mismo- (García, 2007, p. 748). La intimidad es el derecho personal del individuo a resguardar para sí mismo todo aquello que no desea exponer ante otro $u$ otros. Dermizaky (2000) sostiene que el derecho a la intimidad, hacen parte de aquellos derechos personalísimos que:

Sustraen a la persona de la publicidad o de otras perturbaciones a la vida privada, el cual está limitado por las necesidades sociales y los intereses públicos, que particularmente con la informatización de la sociedad y la aparición social de internet, ha traído grandes debates en el mundo jurídico. (p. 218)

La prerrogativa del derecho a la intimidad plantea un paralelo entre sus dos bases: en lo íntimo y en la intimidad. La primera noción refiere todo aquello que se desea realizar en la esfera personalísima del individuo y la segunda a aquello que no se desea dar a conocer a terceros por pertenecer a dicha esfera. Converge con el olvido como un derecho, pues comprometeeseespectro deaquellas acciones e información producida en lo íntimo o que pertenece a la esfera de la intimidad. No se puede soslayar que los derechos fundamentales surgen como mecanismos subjetivos de limitación al poder político, superando la mera delimitación objetiva que, bajo un conjunto de normas de variadas jerarquías, constituye el marco de actuación de las autoridades (Díez-Picazo, 2013, p. 27). Así, los derechos fundamentales conforman un sistema que funciona articulado a la dignidad humana y delimita el ejercicio del poder público, donde con la protección o afectación a algunos de estos, se generan iguales o similares efectos en otros, como sucede con la salud y la vida en los aspectos expuestos ut supra. Cobran así relieve entre aquellas prerrogativas y facultades atadas al ser, reclaman especial importancia por la cadena de efectos deletéreos que generan en la humanidad una vez amenazados, vulnerados o desconocidos, demandando desde su categoría de fundamentales su autonomía e invitando imperativamente al reconocimiento y disposición de rutas y mecanismos expeditos para su protección.

\section{Fundamentalidad autónoma del derecho al olvido. Una apuesta en la justicia digital}

La justicia digital hace alusión a una dimensión metodológica e instrumental de la función pública de administrar justicia. Se trata de la implementación de todos los medios tecnológicos que faciliten la comunicación, la interacción y, en general, la gestión de los trámites jurisdiccionales. Se materializa especialmente en la transición del dossier físico a uno inmaterial, digital. Potencia el acceso a la información y diluye las barreras físicas a través de su gestión desde plataformas digitales (Gascón, 2010, p. 3).

La transición de la justicia en esta esfera contribuye de forma eficaz a materializar 
celeridad y economía, a más de generar claridad, proximidad y transparencia (Bueno, 2010 , p. 3). Incluso, la justicia digital permite que el operador judicial y las autoridades, en general, conozcan de primera mano las formas de relacionamiento digital, sus favores y sus bemoles, de cara a lograr raciocinios adecuados y coherentes con las problemáticas que se generan en una sociedad que se relaciona de manera preferente a través de la red y sus diversos mecanismos y dispositivos. La inclusión digital forma parte del tan necesario desarrollo social, por tal razón, Londoño (2010) plantea que "las TIC (Tecnologías de la Información y las Comunicaciones) se han convertido rápidamente en un conjunto de herramientas indispensables para casi cualquier actividad" (p. 125). Sin embargo, la publicación y difusión de la información contenida en el proceso judicial también genera un halo de efectos nocivos si a la par de la implementación y uso de las tecnologías no se tienen en cuenta los elementos suficientes de protección a los derechos de las personas titulares de la información que se gestiona de forma digital (García, 2007). En este halo de afectaciones cobra valor protagónico la noción de rectificación, en un alcance restringido, y de olvido, en un sentido amplio, conteniéndose el primero en el segundo de los antes anotados.

La volatilidad de la información abiertamente dispuesta en los expedientes digitales, en las providencias judiciales, en el cruce de comunicaciones entre los intervinientes y en los boletines de prensa, entre otras actuaciones más, hace necesario el reconocimiento 0 la reivindicación, si se quiere, del derecho a ser olvidado. No como una prerrogativa absoluta, porque ello significaría la negación de otros derechos ligados al sistema de los fundamentales o de cualquier otra categoría, sino como una limitante subjetiva del poder público susceptible de ser valorado racional- mente ante la tensión o contacto con otros de sus homólogos. Máxime si se tiene en cuenta que la actual sociedad reticular ha enrostrado evoluciones vertiginosas que contraen la obsolescencia temprana de los sistemas de almacenamiento y con esta, la vulnerabilidad de sus mecanismos de seguridad. Solo a manera de ejemplo se puede mencionar que a partir de la entrada en vigencia de las Leyes 1437 de 2011 y 1564 de 2012, la justicia digital acentúa su tono tenue incorporado desde 1996 con la Ley 270 de esa anualidad; sin embargo, el denominado Plan de Justicia Digital ha sido una verdadero naufragio, cuyo fracaso se evidenció al producir la parálisis del sistema judicial a partir del mes de marzo de 2020, con la coyuntura multidimensional y mundial que generó la COVID-19, indicando que el sistema judicial colombiano no evoluciona a la velocidad que exigen las necesidades sociales.

Por otra parte, la ubicuidad de la información, dada la alta velocidad de propagación de la información expuesta en las redes informáticas, contrae consecuencias y respuestas inmediatas y precisamente, como se acaba de mencionar ut supra, esta es una característica con la que no cuentan los mecanismos jurisdiccionales de protección y hacen necesario extremar las precauciones y medidas preventivas, que cobran mayor valor y criterios objetivos de procedimiento bajo el manto del derecho fundamental al olvido.

La autonomía brinda esa capacidad para que el derecho al olvido pueda invocarse sin extensiones innecesarias que hagan su aplicabilidad tardía, compleja y confusa. No en vano Leturia (2016) propone que "el derecho al olvido sea tomado como un derecho fundamental desde su lógica y los conflictos que este genera para ser justificado en el refuerzo de la privacidad y otros derechos" (p. 97). Por su parte, Alexy (1993), en la teoría de los 
derechos fundamentales, plantea que "una de las características de los derechos fundamentales son los conflictos y contraposiciones que genera" (p. 45).

Si bien este derecho no impide el desarrollo de la publicación de la información en los procesos judiciales, genera reales garantías que limitan el uso de la información privada que se encuentran dentro del expediente digital, que radican en la libertad y difusión de la misma. "Las libertades fundamentales de expresión y de acceso a las informaciones en la red, deben leerse de manera proporcionada y no en contradicción con el derecho a la protección de datos personales" (Galvis y Salazar, 2019 , p. 49). Dicho de otra forma, refiere a la posibilidad de ser garante como derecho fundamental, desde diferentes mecanismos jurídicos y extrajurídicos - procesales y extraprocesales- existentes, de conformidad con los criterios para su reconocimiento.

\section{Conclusión}

En una sociedad que se relaciona de forma preferente y progresiva a través de mecanismos y dispositivos tecnológicos, la información vertida en esa volatilidad que presuponen las redes informáticas y telemáticas es un elemento axial de la puesta en escena jurídica de algunas condiciones inherentes a la esencia humana. Además, en la denominada justicia digital, esto es, en el ejercicio de la función pública de impartir justicia judicial a través de medios tecnológicos, se incrementan los riesgos a merced de la cantidad y de la calidad de la información que de los intervinientes y de terceros se ventila en el decurso procesal. En este escenario, el derecho a que esa información sea eliminada 0 anonimizada cobra relieve protector de aquella característica definitoria de la humanidad que se arraiga en su percepción como fin y nunca como un instrumento para consecución de otros fines: la dignidad humana.

En ese sentido, universalmente se ha aceptado que existen derechos que, dada su importancia asociada al mantenimiento y al desarrollo prospectivo de la humanidad, gozan de especiales garantías cimentadas en la denominación de fundamentales y que, en razón de dicha condición, comprometen mecanismos jurídicos preferentes e idóneos frente a otros derechos. De ahí que el derecho a ser olvidado constituye, en una sociedad tecnológica desarrollada a partir del insumo de la información personal, en esencia, un derecho fundamental que no puede ni debe depender de la conexión con otros derechos para su protección, como se ha considerado hasta ahora. Sino que, por el contrario y en lógica de avance prospectivo del desarrollo humano, los mecanismos de acceso y exigencia deben corresponder con la importancia que esta prerrogativa comporta frente a las nuevas dinámicas humanas, especialmente en la litigación digital y sus procedimientos accesorios. Se trata del reconocimiento del olvido como un derecho fundamental y, por consiguiente, autónomo, que arraiga dicha categoría en su relación inescindible con la condición humana y el mantenimiento de la misma en la era tecnológica. 


\section{Referencias}

Alexy, R. (1993). Teoría de los derechos fundamentales (E. Garzón, trad.). Centro de Estudios Constitucionales. (Original publicado en 1986 con el título Theorie der Griindrechte).

Arango, A. (2005). El concepto de derechos sociales fundamentales. Legis.

Bueno, F. (2010). E-justicia: hacia una nueva forma de entender la justicia. Riedpa: Revista Internacional de Estudios de Derecho Procesal y Arbitraje, (1), 1-10. https:// dialnet.unirioja.es/ descarga/articulo/3700453.pdf

Coll, A. (2018). La discrecionalidad administrativa laboral. Existencia, otorgamiento, ejercicio y control judicial neoconstitucionales. Prolegómenos, 21(42), 73-90. http:// www.scielo. org.co/scielo.php?script=sci_arttext $\&$ pi$d=S 0121-182 \times 2018000200073$

Coll, A. y González, D. (2020). La dignidad humana y la defensa a ultranza del derecho a la vida. Un debate entre lo ético, lo jurídico y lo humano. En A. Coll y R. Restrepo (comps.), Derecho constitucional, penal y privado. Análisis de actualidad (pp. 73-116). Danaleja.

Congreso de la República (18 de enero de 2011). Ley 1437 de 2011. Por la cual se expide el Código de Procedimiento Administrativo y de Io Contencioso Administrativo. Diario Oficial No. 47.956. https:// www.icbf.gov.co/cargues/avance/docs/ley_1437_2011.htm

Congreso de la República (12 de julio de 2012). Ley 1564 de 2012. Por medio de la cual se expide el Código General del Proceso y se dictan otras disposiciones. Diario Oficial No. 48489. http:// www.suin-juriscol.gov.co/ viewDocument.asp?ruta=Leyes $/ 1683572$

Congreso de la República (15 de marzo de 1996). Ley 270 de 1996. Estatutaria de la Administración de Justicia. Diario Oficial No. 42.745. http:// www.secretariasenado.gov.co/ senado/basedoc/ley_0270_1996.html
Corte Constitucional Colombiana (3 de agosto de 1998). Sentencia T-395/98 (Alejandro Martínez Caballero, M. P.). https:// bit.ly/ 3q1y2p7

Corte Constitucional Colombiana (25 de mayo de 2000). Sentencia T-1306/00 (Fabio Morón Díaz, M. P.). https:// www.corteconstitucional.gov.co/ relatoria/2000/ T-1306-00.htm

Corte Constitucional Colombiana (22 de enero de 2007). Sentencia T-016/07 (Humberto Antonio Sierra Porto, M. P.). https:// www.corteconstitucional.gov.co/ relato$\mathrm{ria} / 2007 / \mathrm{T}-016-07 . \mathrm{htm}$

Corte Constitucional Colombiana (31 de julio de 2008). Sentencia T-760/08 (Manuel José Cepeda Espinosa, M. P.). https:// www.corteconstitucional.gov.co/relatoria/2008/t-760-08.htm

Chéliz, I. (2016). El "derecho al olvido digital". Una exigencia de las nuevas tecnologías, recogida en el futuro reglamento general de protección de datos. Actualidad Jurídica Iberoamericana, (5), 255-271.

Dermizaky, P. (2000). El derecho a la intimidad. Ius et Praxis, 6(1), 177-193. https:// www.redalyc.org/pdf/ 197/ 19760113.pdf

Díez-Picazo, L. (2013). Sistema de derechos fundamentales. Thomson Civitas.

Ferrajoli, L. (2006). Sobre los derechos fundamentales. Cuestiones Constitucionales, (15), 113-136. http:// www.scielo.org.mx/pdf/ cconst/n15/ 1405-9193-cconst-15-113.pdf

Galvis, L. y Salazar, L. (2019). Alcance del derecho al olvido en el tratamiento de datos personales en Colombia. Verba luris, 14(41), 45-63. https:// bit.ly/ 3zaJYc6 
García, A. (2007). La protección de datos personales: derecho fundamental del siglo XXI. Un estudio comparado. Boletín Mexicano de Derecho Comparado, 40(120), 743-778. http:// www.scielo.org. mx/pdf/bmdc/v40n120/ v40n120a3.pdf

Garzón, E. (2016). Derechos innominados en el sistema interamericano. Dixi, 18(24). https:/ / dialnet.unirioja.es/ servlet/ autor?codigo $=3985446$

Gascón, I. (2010). La e-Justicia en la Unión Europea: Balance de situación y planes para el futuro (en diciembre de 2009). En C. Senés (dir.), Presente y futuro de la e-justicia en España y la Unión Europea (pp. 83-125). Universidad Complutense de Madrid.

Guasch, V. y Soler, J. (2015). El interés legítimo en la protección de datos. Revista de Derecho UNED, 16, 417-438. http:// revistas.uned.es/index.php/RDUNED/article/ view/ 15245/ 13350

Kant, I. (1875). Fundamentación de la metafísica de las costumbres. Escuela de Filosofía Universidad ARCIS. https://pmrb.net/books/ kantfund/fund_metaf_costumbres_vD.pdf

Leturia, F. (2016). Fundamentos jurídicos del derecho al olvido: ¿un nuevo derecho de origen europeo o una respuesta típica ante colisiones entre ciertos fundamentos? Revista Chilena de Derecho, 43(1), 91-113. https:// scielo.conicyt.cl/scielo.php?pi$d=$ S0718-34372016000100005\&script=sci_arttext\&tlng=en

Londoño, N. (2010). El uso de las TIC en el proceso judicial: una propuesta de justicia en línea. Revista de la Facultad de Derecho y Ciencias Políticas, 40(112), 123-142. https:// revistas. upb.edu.co/index.php/derecho/article/ view/3775

de Lucas, J. (2008). La extensión de los agentes del pluralismo (Vol. 4). Eusko Jaurlaritzaren Agitalpen-Zerbitzu Nagusia.
Marshall, P. (2017). Clasificación de los derechos fundamentales. En P. Contreras y C. Salgado (coords.), Manual de derechos fundamentales: Teoría general (pp. 93-118). Universidad Austral de Chile.

Mieres, L. (2014). El derecho al olvido digital. Documento de Trabajo 186/2014 - Laboratorio de Alternativas, 41, 1-57. https://d3cra5ec8gdi8w.cloudfront. net/ uploads/ documentos/2014/06/10/documentos_1862014revisado_6f969637.pdf

Moreno, U. y Esponda, C. (2008). El concepto de autonomía en la fundamentación de la metafísica de las costumbres de I. Kant. Cuadrante Phi, (17), 1-13. https:// javeriana.edu.co/ cuadrantephi/pdfs/ N.17/ 4.\%20Kant.pdf 